\title{
Possible Implication of Hypoxia-mediated Incomplete Neutrophil Extracellular Trap Formation in Pneumonia-induced Exacerbation of Lung Diseases
}

\author{
Sakiko Masuda \\ Hokkaido University \\ Kurumi Kato \\ Hokkaido University \\ Misato Ishibashi \\ Hokkaido University \\ Yuka Nishibata \\ Hokkaido University \\ Ayako Sugimoto \\ Hokkaido University \\ Daigo Nakazawa \\ Hokkaido University \\ Satoshi Tanaka \\ Hokkaido University \\ Utano Tomaru \\ Hokkaido University \\ Ichizo Tsujino \\ Hokkaido University \\ Akihiro Ishizu ( $\nabla$ aishizu@med.hokudai.ac.jp ) \\ Hokkaido University
}

\section{Research Article}

Keywords: Neutrophil extracellular traps, Hypoxia, DNase I, Actin

Posted Date: April 9th, 2021

DOI: https://doi.org/10.21203/rs.3.rs-388216/v1

License: (9) This work is licensed under a Creative Commons Attribution 4.0 International License.

Read Full License 
Page 2/24 


\section{Abstract}

When patients with preexisting lung diseases, such as chronic obstructive pulmonary disease, interstitial pneumonitis, and pulmonary arterial hypertension, develop pneumonia, the complication often exacerbates the underlying diseases. Although neutrophil extracellular traps (NETs) are important components of innate immune system, the residue of NETs in the tissue can harm the host. We examined the expression of hypoxia-inducible factor $1 \mathrm{a}(\mathrm{HIF}-1 \mathrm{a})$ and NETs in the lungs of patients with lung diseases complicated with pneumonia, and investigated the properties of NETs generated under hypoxia. This study demonstrated that the amount of NETs in pulmonary lesions was greater in patients with pneumonia than in patients without pneumonia and displayed a positive correlation between the amount of NETs and HIF-1a expression. We further demonstrated that the formation of typical lytic NETs was suppressed and round-shaped NETs were generated under hypoxic conditions in vitro. These round NETs were resistant to digestion by the principal NET regulator, DNase I. Focusing on actin rearrangement in neutrophils stimulated under hypoxic conditions, we found that G-actin polymerization and F-actin degradation-both of which are observed time-dependently under normoxic conditions-were disrupted, suggesting that hypoxia mediated the incomplete NET formation. Moreover, neutrophils stimulated under hypoxic conditions possessed cytotoxicity. Accumulation of neutrophils that form degradation-resistant NETs and possess cytotoxicity, which are generated under hypoxic circumstances, are expected to be involved in exacerbation of underlying lung diseases complicated with pneumonia.

\section{Introduction}

Globally, 450 million people suffer from pneumonia caused by bacterial, viral, or fungal infection, and 4 million of those die of the disease every year ${ }^{1}$. Streptococcus pneumonia, Haemophilus influenza, and Staphylococcus aureus are common pathogens of pneumonia ${ }^{2}$. When pneumonia occurs in patients with preexisting lung diseases, such as chronic obstructive pulmonary disease (COPD), the complication often exacerbates the underlying diseases. Bacterial and viral infections are reported to be risk factors for exacerbation of $\mathrm{COPD}^{3,4}$. Patients with pulmonary hypertension $(\mathrm{PH})$ are apt to develop pneumonia, and $7 \%$ of $\mathrm{PH}$ patients die from pneumonia ${ }^{5}$. In these diseases, there is insufficient distribution of oxygen to the peripheral lung tissues because of obstructions of the bronchi or because pulmonary microcirculation is disturbed by the localized hypertension, resulting in hypoxemia. Pneumonia also induces hypoxemia, and it is considered that serious hypoxia occurs in the lungs when preexisting lung diseases are complicated with pneumonia.

Neutrophils play an important defensive role at the forefront of invasion of pathogenic microorganisms and they possess diverse antimicrobial functions, including phagocytosis, production of reactive oxygen species (ROS), and release of neutrophil extracellular traps (NETs). NETs are composed of web-like extracellular DNA decorated with antibacterial substances, such as neutrophil elastase (NE), myeloperoxidase (MPO), and matrix metalloproteinases (MMPs), and are able to trap and eliminate pathogenic microorganisms ${ }^{6}$. The formation of NETs by the potent NET inducer, phorbol myristate 
acetate (PMA), is dependent on ROS production by the activation of NADPH oxidase via the protein kinase $C$ and Raf-MEK-ERK signaling pathways ${ }^{7,8}$. It has been suggested that ROS translocates peptidylarginine deiminase 4 (PAD4) from the cytoplasm to the nucleus ${ }^{9}$. In the nucleus, PAD4 citrullinates the arginine residue of histone $\mathrm{H} 3$ and alters its molecular conformation, resulting in the detachment of DNA that coils around histones ${ }^{10,11}$. In addition, NE and MPO contained in azurophilic granules of neutrophils are degranulated and released into the cytoplasm and then move to the nucleus $^{12}$. These steps also occur in a ROS-dependent manner. In the nucleus, NE synergizes with MPO, and these molecules cleave histones to promote DNA unraveling ${ }^{13}$. Eventually, the unraveled DNA breaks through the nuclear membrane, followed by mixing with cytoplasmic antimicrobial substances. NE also cleaves gasdermin $D$ to generate its active form, which makes pores in the cell membrane ${ }^{14}$, and finally the unraveled DNA decorated with antibacterial substances emerges from the cell membrane ${ }^{15}$. The common pneumonia pathogens, including Streptococcus pneumonia, Haemophilus influenza, and Staphylococcus aureus induce NETs ${ }^{8}$.

The formation of NETs in lung diseases has been reported. The spontaneous formation of NETs has been observed in the sputum and peripheral blood from COPD patients ${ }^{16-18}$. The expression of PAD4, which is an essential enzyme for the generation of NETs, is increased in the lungs of COPD patients ${ }^{19}$. Moreover, it has been demonstrated that increased NET formation in the airways is associated with increased severity of $\mathrm{COPD}^{20}$. The presence of NET-forming neutrophils in occlusive plexiform vessels and intrapulmonary thrombi in patients with $\mathrm{PH}$ has also been shown ${ }^{21}$. These findings suggest that abundant NETs are formed in the pulmonary lesions of patients with preexisting lung diseases complicated with pneumonia.

Although NETs are important elements of innate immunity, excessive NETs are sometimes burdensome in terms of their cytotoxicity ${ }^{22}$. Therefore, we hypothesized that the large amount of NETs formed in the pulmonary lesions of preexisting lung diseases complicated with pneumonia could be involved in the exacerbation of the underlying diseases. However, there is conflicting evidence that needs to be addressed. It has been demonstrated that NET formation is reduced when neutrophils are stimulated by PMA under hypoxic conditions ${ }^{23}$. Decreased formation of NETs under hypoxic conditions was also observed when neutrophils were stimulated by Mycobacterium tuberculosis ${ }^{24}$. Because the pathway to NET formation depends on the ROS production, it seems to be reasonable that NET formation is suppressed by the decrease in oxygen supply under hypoxia. In contrast, the net amount of NETs is defined by the balance between their generation and degradation. Because of the adverse effects on the hosts, NETs are physiologically degraded by DNase I in the plasma ${ }^{25}$. However, it has not been determined how hypoxia influences the degradation of NETs.

In this study, we first assessed the hypoxic state of the lungs by monitoring hypoxia-inducible factor $1 \mathrm{a}$ (HIF-1a) expression using formalin-fixed, paraffin-embedded (FFPE) sections from patients with lung diseases, pneumonia, or both. It has been demonstrated that the expression of HIF-1a is induced by 
tissue hypoxia ${ }^{26}$. Next, we determined the amount of NETs in these lung tissues. We also examined the influence of hypoxic conditions on NET generation and degradation in vitro.

\section{Material And Methods}

\section{Human materials}

FFPE tissues of normal lungs $(n=2)$ and disease-affected lungs $(n=21)$ were used. The affected lung tissues were obtained from patients with pneumonia $(n=7)$, lung diseases without pneumonia $(n=9)$, and preexisting lung diseases complicated with pneumonia $(n=5)$. The lung diseases included COPD, interstitial pneumonitis (IP), pulmonary arterial hypertension (PAH), COPD with $\mathrm{PH}$, and IP with $\mathrm{PH}$ (Table S1). All samples were obtained by autopsy. In each case, written informed consent for the use of FFPE lung tissues was obtained from a representative of the bereaved family. Peripheral blood was obtained from healthy volunteers after acquisition of written informed consent. This study was conducted with the permission of the Ethics Committee of the Faculty of Health Sciences, Hokkaido University (Permission Nos. $18-7$ and 18-34). This study was in adherence with the Declaration of Helsinki.

\section{Immunohistochemistry (IHC) for HIF-1a}

FFPE lung sections were reacted with anti-HIF-1a antibody (clone mgc3, mouse IgG1, 1:800 dilution; Abcam, Cambridge, MA, USA) or isotype control mouse IgG1 (BD, Franklin Lakes, NJ, USA) for 1 hour at room temperature (RT). After washing with phosphate-buffered saline (PBS), the sections were next reacted with horseradish peroxidase (HRP)-conjugated goat anti-mouse IgG1 antibody (1:50 dilution; KPL, Gaithersburg, MD, USA) for 1 hour at RT. HRP activity on the sections was detected by 3,3'diaminobenzidine substrates (Nichirei Biosciences, Tokyo, Japan) followed by counterstaining with Mayer's hematoxylin dye.

\section{Immunofluorescent staining for NETs}

FFPE lung sections were reacted with anti-citrullinated histone $\mathrm{H} 3$ (Cit H3) antibody (polyclonal rabbit IgG, 1:100 dilution; Abcam) and anti-MPO antibody (polyclonal goat IgG, 1:200 dilution; R\&D Systems, Minneapolis, MN, USA) for 1 hour at RT. For controls, rabbit IgG (Abcam) and 10\% goat serum (Nichirei Biosciences) were used. After washing with PBS, the sections were next reacted with Alexa Fluor 594conjugated donkey anti-rabbit IgG antibody (1:500 dilution; Abcam) and Alexa Fluor 488-conjugated donkey anti-goat IgG antibody (1:500 dilution; Abcam) for 1 hour at RT. The sections were finally mounted with a mounting solution containing 4',6-diamidino-2-phenylindole (DAPI; Vector Laboratories, Burlingame, CA, USA).

\section{Induction and degradation of NETs}

Induction and degradation of NETs were carried out as described previously ${ }^{27,28}$. Briefly, peripheral blood neutrophils from healthy volunteers obtained using Polymorphprep (Axis-Shield, Dundee, Scotland) were 
suspended in RPMI 1640 medium containing $10 \%$ fetal bovine serum (FBS; $5 \times 10^{5} / \mathrm{mL}$ ), seeded in chambers of 4-well chamber slides, and then pre-incubated for 30 minutes at $37^{\circ} \mathrm{C}$. The cells were exposed to 20 or $100 \mathrm{nM}$ PMA for 4 hours at $37^{\circ} \mathrm{C}$ to induce NETs under normoxic $\left(21 \% \mathrm{O}_{2}\right)$ or hypoxic $\left(1 \% \mathrm{O}_{2}\right)$ conditions. The partial pressure of oxygen $\left(\mathrm{PO}_{2}\right)$ was set according to the literatures ${ }^{29,30}$. To create hypoxic culture conditions, Bionix Hypoxic Cell Culture Kit (Sugiyama-gen, Tokyo, Japan) was used. The $\mathrm{PO}_{2}$ was monitored by an oximeter. DNase I treatment for digestion of NETs was performed after NET induction. NETs were exposed to $10 \mathrm{U} / \mathrm{mL}$ DNase I (Invitrogen, Waltham, MA, USA) for 30 minutes at $37^{\circ} \mathrm{C}$.

\section{Detection of cell-free double-stranded DNA (dsDNA) in supernatants}

Peripheral blood neutrophils $\left(5 \times 10^{5} / \mathrm{mL}\right)$ were treated with $20 \mathrm{nM}$ PMA for 4 hours at $37^{\circ} \mathrm{C}$ to induce NETs under normoxic $\left(21 \% \mathrm{O}_{2}\right)$ or hypoxic $\left(1 \% \mathrm{O}_{2}\right)$ conditions. Supernatants were collected by centrifugation, and then the concentrations of dsDNA in the supernatants were determined by using Quanti-iT dsDNA Assay Kit (Life technologies, Eugene, OR, USA).

\section{Fluorescent staining for actin}

NET-forming neutrophils were fixed with $4 \%$ paraformaldehyde for 15 minutes and then permeated with $0.5 \%$ Triton-X 100 for 5 minutes at RT. Thereafter, the cells were reacted with anti- $\beta$ actin antibody (clone mAbcam 8226, mouse IgG1, 1:100 dilution; Abcam) or isotype control mouse IgG1 (Abcam) at $4^{\circ} \mathrm{C}$ overnight. After washing with PBS, the cells were next reacted with Alexa Fluor 488-conjugated goat antimouse IgG1 antibody (1:500 dilution, Abcam) and $100 \mathrm{nM}$ Acti-stain 555 phalloidin (Cytoskeleton, Denver, CO, USA) for 30 minutes or 1 hour. In cells, actin exists in two different forms: non-polymerized granular form, G-actin, and polymerized filamentous form, F-actin. The anti- $\beta$ actin antibody recognizes G-actin ${ }^{31}$, whereas phalloidin binds specifically to F-actin. The cells were finally mounted with the mounting solution containing DAPI.

\section{Inhibition of actin polymerization}

To inhibit actin polymerization, $0.5 \mu \mathrm{M}$ cytochalasin D (CyD; Cayman Chemical, Ann Arbor, MI, USA) was added 30 minutes before or after, or simultaneously with administration of PMA (20 nM). The concentration of CyD followed that of a previous study ${ }^{32}$. After 4 hours incubation, NET formation was evaluated by fluorescent staining with DAPI.

\section{Cytotoxic assay}

Peripheral blood neutrophils $\left(5 \times 10^{5} / \mathrm{mL}\right)$ were treated with $20 \mathrm{nM}$ PMA for 4 hours at $37^{\circ} \mathrm{C}$ to induce NETs under normoxic $\left(21 \% \mathrm{O}_{2}\right)$ or hypoxic $\left(1 \% \mathrm{O}_{2}\right)$ conditions. The supernatants were diluted 2 - or 4 -fold with culture medium. Normal human lung fibroblasts (NHLF; Lonza, Tokyo, Japan) were cultured in DMEM containing $10 \% \mathrm{FBS}$ at $37^{\circ} \mathrm{C}$. NHLF were seeded in wells of 96 -well plates $\left(5 \times 10^{4} / \mathrm{mL}\right)$. After 24 hours pre-incubation, the supernatants were added to the NHLF culture and incubated for another 24 
hours at $37^{\circ} \mathrm{C}$. Cell viability of NHLF was calculated with Cell Counting Kit-8 (Dojindo Laboratories, Kumamoto, Japan). The concentration of MMP-9 in the supernatants was determined by a human MMP9 enzyme-linked immunosorbent assay (ELISA) kit (Proteintech, Deansgate Manchester, UK).

\section{Data reproducibility}

All in vitro experiments were repeated independently, and the reproduction of data was confirmed.

\section{Statistical analysis}

One-way analysis of variance followed by Tukey-Kramer post hoc test was applied for comparison within multiple groups. Mann-Whitney U-test was applied for comparison between two non-parametric groups. $P<0.05$ was considered as statistically significant.

\section{Results}

\section{Enhanced expression of HIF-1a in the lungs by complication of pneumonia with preexisting lung diseases}

To elucidate the hypoxic state in the lungs, IHC for HIF-1 a was carried out using FFPE tissues from normal lungs (Group 1, $n=2$ ), from lungs with pneumonia (Group 2, $n=7$ ), lung diseases without pneumonia (Group 3, $n=9$ ), and preexisting lung diseases with pneumonia (Group 4, $n=5$ ). Details of the lung tissue samples are listed in Table S1. HIF-1a was expressed in bronchial epithelial cells, inflammatory cells, and alveolar macrophages (Figure S1a). The specificity of IHC was guaranteed by the negative staining for isotype control mouse IgG1 (Figure S1b). Because there was no obvious difference in expression level between cell types, we focused on bronchial epithelial cells. After observing the whole section under a microscope, the expression level was scored by a cytoscreener (with 10 years of experience) as follows: 0 , undetectable; 1 , weak; 2 , moderate; and 3 , strong (Fig. 1a), and then the scoring was confirmed by a pathologist (with 25 years of experience). This is a standard process of clinical cytoscreening. HIF-1a expression in Group 4 was significantly higher than in Groups 2 and 3 (Fig. 1b). These findings corresponded to our prediction that serious hypoxia occurs in the lungs when preexisting lung diseases are complicated with pneumonia.

\section{Increase in NETs in the lungs by complication of pneumonia with preexisting lung diseases}

We next determined the amount of NETs in the lung tissues of the 4 groups (serial sections of FFPE specimens used in Fig. 1). NETs were recognized as $\mathrm{Cit}^{\mathrm{H} 3^{+}}$substances mixed with DNA and MPO in the alveoli, bronchi, and small vessels. The amount of NETs was semi-quantified by the cytoscreener as follows: Score 0, unrecognizable; Score 1, scattered small NETs; Score 2, scattered large NETs; and Score 3 , dense large NETs, and then the scoring was confirmed by the pathologist. NETs were detected in Groups 2, 3, and 4 but not Group 1 (Fig. 2a). The amount of NETs was significantly higher in Groups 2 and 4 than in Groups 1 and 3, respectively (Fig. 2b). These findings are consistent with our prediction of increased NETs in pulmonary lesions of patients with preexisting lung diseases after complication of 
pneumonia. There was a significant positive correlation between HIF-1a expression score and amount of NETs (Fig. 2c).

\section{Neutrophils stimulated by PMA under hypoxic conditions}

When peripheral blood neutrophils were stimulated by PMA under hypoxic conditions $\left(1 \% \mathrm{O}_{2}\right)$, numerous round substances of a size larger than non-treated neutrophils were observed, and the typical lytic NETs generated under normoxic conditions $\left(21 \% \mathrm{O}_{2}\right)$ did not appear (Fig. 3a). These round substances were also generated under normoxic conditions in fewer numbers than under hypoxic conditions. Although round-shaped nuclei have been shown as characteristics of apoptotic neutrophils ${ }^{33}$, the round substances were regarded as NET-forming neutrophils because of the positive staining for Cit H3, MPO, and DNA.

To confirm this, concentrations of dsDNA in the supernatants were determined. The levels of dsDNA in the supernatants were significantly elevated after PMA stimulation under hypoxic conditions as well as under normoxic conditions (Fig. 3b), suggesting that the cell membrane of neutrophils was perforated and that decondensed DNA was exuded from the cytoplasm. The increase in dsDNA in the supernatants remained at $40 \%$. This corresponded with the finding that all neutrophils exposed to PMA did not undergo NET formation. The NET-forming neutrophils appear to be in a state before releasing web-like DNA; thus, this is consistent with previous reports of reduced NET formation under hypoxia ${ }^{23,24}$. In this study, such round-shaped NETs formed by neutrophils in the state before releasing web-like DNA are called "round NETs" (Figure S2).

Next, we investigated whether NETs generated under normoxic $\left(21 \% \mathrm{O}_{2}\right)$ and hypoxic $\left(1 \% \mathrm{O}_{2}\right)$ conditions could be degraded by the principal NET degrader, DNase I (Fig. 3c). The lytic NETs generated under normoxic conditions were completely degraded by DNase I. In contrast, round NETs generated under both normoxic and hypoxic conditions remained even after DNase I treatment.

As shown in Figs. 3a and 3c, the neutrophils without PMA stimulation were apt to detach from the slides. To compare the digestion of NETs by DNase I, five photographs were taken randomly before and after DNase I treatment, and then mean nuclei area was calculated using Image $J$ 1.50i software (http://allpcworld.com/download-imagej-1-50i-free/) as follows: DAPI ${ }^{+}$areas/number of neutrophils (pixels). Image analysis demonstrated that NETs induced under normoxic conditions were partly but significantly degraded by DNase I but NETs induced under hypoxic conditions were not degraded by DNase I (Fig. 3d). These findings suggest that lytic NETs are sensitive to DNase I, whereas round NETs are resistant to DNase I. A similar phenomenon-the DNase I-resistance of round-shaped NETs induced by PMA with propylthiouracil-has been reported previously ${ }^{34}$.

\section{Disruption of actin rearrangement during NET formation under hypoxic conditions}

Morphological change in cells results from a rearrangement of the cytoskeleton. To assess the cytoskeletons of neutrophils during NET formation, we focused on actin rearrangement. It has been 
reported that digestion of F-actin by NE, which occurs ROS-dependently, is required for NET formation ${ }^{12}$. However, another study has indicated that ROS-dependent actin polymerization-transformation from Gactin to F-actin-is essential for NET formation ${ }^{35}$. To resolve this conflict, we utilized fluorescent staining to follow chronological changes in actin during NET formation by using an anti- $\beta$ actin antibody that binds to G-actin ${ }^{31}$ and phalloidin that binds to F-actin specifically (Fig. 4a).

Under normoxic conditions $\left(21 \% \mathrm{O}_{2}\right)$, G-actin was detected most strongly 30 minutes after PMA stimulation and then gradually decreased thereafter (Fig. 4b). F-actin was increased as G-actin was decreased and peaked 1-2 hours after PMA stimulation. The amount of F-actin was also decreased afterward. Finally, both signals became almost undetectable in lytic NETs observed 4 hours after PMA stimulation. These findings are consistent with the previous report of the absence of actin in NETs ${ }^{36}$.

Next, to elucidate actin rearrangement during NET formation under hypoxic conditions $\left(1 \% \mathrm{O}_{2}\right)$, we examined the distributions of G-and F-actins after 4 hours incubation with PMA and compared them with the findings under normoxic conditions $\left(21 \% \mathrm{O}_{2}\right.$; Fig. $\left.4 \mathrm{c}\right)$. As observed in the former experiment, neither $\mathrm{G}$ nor F-actin was evident in lytic NETs generated under normoxic conditions, although there were scattered actins around the cells. Under hypoxic conditions, in contrast, both G- and F-actins were detected in round NETs, especially in the periphery of the cells. We consider this finding to be the "actin caps" that have been recognized in neutrophils under hypoxia ${ }^{37}$.

These findings suggest that during NET formation under normoxic conditions, polymerization of G-actin occurs in an earlier period (30 minutes to 1 hour after PMA stimulation), and degradation of F-actin follows in a later period ( 1 to 4 hours after PMA stimulation). Hypoxia appears to disrupt the actin rearrangement that leads to the formation of lytic NETs after PMA stimulation.

\section{Suppression of NET generation by actin polymerization inhibitor}

To examine the association between the generation of NETs and actin polymerization, we next performed experiments using an actin polymerization inhibitor, CyD. CyD was added to peripheral blood neutrophils simultaneously with PMA, or 30 minutes before or after the addition of PMA, and then NET formation was monitored by DNA staining with DAPI after 4 hours incubation under normoxic conditions $\left(21 \% \mathrm{O}_{2}\right.$; Figure S3a). When neutrophils were pre-treated with CyD, the generation of NETs was suppressed almost completely. In contrast, the suppression of formation of lytic and round NETs was partial when CyD was administered simultaneously and 30 minutes after PMA stimulation (Figure S3b).

\section{Effects of inhibition of actin polymerization on susceptibility of NETs to DNase I}

We demonstrated that round NETs generated under hypoxic conditions are resistant to DNase I digestion. G-actin, which can bind DNase I, is regarded as a putative inhibitor of DNase $\left.\right|^{25,38,39}$. To investigate the association between the DNase I resistance of round NETs and G-actin, peripheral blood neutrophils were treated simultaneously with CyD and PMA under normoxic conditions $\left(21 \% \mathrm{O}_{2}\right)$. After 4 hours incubation, 
actin distribution was assessed by fluorescent staining. It was noted that G-actin remains in round NETs (Fig. 5a), which is a result similar to that when neutrophils are treated with PMA under hypoxic conditions $\left(1 \% \mathrm{O}_{2}\right.$; see Fig. 4c). These findings suggested that the inhibition of actin polymerization by CyD under normoxic conditions suppressed the formation of lytic NETs, resulting in the increase in round NETs; thus, this mimicked NET formation under hypoxic conditions.

We further determined the DNase I susceptibility of round NETs that contained G-actin (Fig. 5b). Round NETs induced by PMA with CyD were found to be not digested by DNase l, although lytic NETs induced by PMA without CyD, which did not contain G-actin, was digested completely (Fig. 5c). These findings suggest a possible association between G-actin and the DNase I resistance of round NETs.

\section{Cytotoxic potential of neutrophils stimulated under hypoxic conditions}

Finally, we examined whether neutrophils stimulated under hypoxic conditions exhibited cytotoxicity. The supernatants of neutrophils stimulated under normoxic $\left(21 \% \mathrm{O}_{2}\right)$ and hypoxic $\left(1 \% \mathrm{O}_{2}\right)$ conditions were applied to a culture of NHLF. Twenty-four hours later, the viability of the cells was determined with a cell counting kit. Compared to the supernatants from non-treated neutrophils and medium containing equivalent concentrations of PMA, the number of viable NHLF was reduced significantly by the supernatants of neutrophils stimulated under hypoxic conditions similarly to those under normoxic conditions (Fig. 6a). These findings suggest that neutrophils stimulated under hypoxic conditions can secrete cytotoxic factors to the same extent as NETs generated under normoxic conditions, despite NETs being incompletely formed under hypoxic conditions.

MMP-9 has been demonstrated to be a critical cytotoxic molecule that is secreted from neutrophils during NET formation ${ }^{40}$. Regardless of $\mathrm{PO}_{2}$, a significant increase in MMP-9 secretion into the supernatants of PMA-stimulated neutrophils was observed (Fig. 6b).

\section{Discussion}

We first demonstrated that expression of HIF-1a in the lungs was increased by pneumonia complicated with preexisting lung diseases. This may be agreeable because pneumonia aggravates the hypoxemia by the underlying lung diseases. In addition, several pneumonia-mediated factors, including growth factors, cytokines, and ROS, can stabilize HIF-1 $1 a^{41,42}$.

We next demonstrated that the amount of NETs in pulmonary lesions was greater in tissues from patients with pneumonia than in those without pneumonia, and there was a positive correlation between the amount of NETs and the expression levels of HIF-1a. Because NETs possess both beneficial and deleterious aspects ${ }^{8}$, we hypothesized that NET-mediated cytotoxicity is involved in the exacerbation of underlying lung diseases complicated with pneumonia. However, preceding studies showed that NET formation was suppressed under hypoxic conditions ${ }^{23,24}$, which is contradictory to our observations of the positive correlation between the HIF-1a expression levels and amount of NETs in the lungs. 
To resolve this conflict, considering that the net amount of NETs is defined by the balance between their generation and degradation, we attempted to clarify the NET generation and degradation under hypoxia. The series of in vitro experiments revealed that (1) the formation of lytic NETs was suppressed and round NETs were generated under hypoxic conditions, (2) round NETs were resistant to digestion by DNase I, (3) actin rearrangement that occurs during NET formation under normoxic conditions is disrupted under hypoxic conditions, and (4) neutrophils stimulated under hypoxic conditions possess cytotoxicity, despite NET formation not being completed.

Rearrangement of the cytoskeleton, especially actin, is critical in the formation of NETs. Figure S4 summarizes the effects of hypoxia on NET formation, including the disruption of actin rearrangement. Because the pathway to NET formation induced by PMA depends on the ROS production ${ }^{7-9}$, it seems reasonable that NET formation is suppressed by the decreased supply of oxygen under hypoxia.

We demonstrated that round NETs generated under hypoxic conditions were resistant to degradation by DNase I. The DNA of round NETs may contain putative molecules that disturb DNase I. Although it has not been determined what molecules confer this degradation resistance on NETs, G-actin is a possible candidate. Kabsch et al. have demonstrated that G-actin can bind to DNase I and inhibit its nuclease activity ${ }^{38}$. Moreover, G-actin has been shown to inhibit degradation of NETs by DNase I in a dosedependent manner ${ }^{25,39}$. Further studies are needed to clarify whether G-actin that remains in neutrophils with the formation of round NETs could affect their susceptibility to DNase I under hypoxic conditions.

We demonstrated that neutrophils stimulated under hypoxic conditions possessed cytotoxic potential similar to NETs generated under normoxic conditions. In this study, NHLF were employed as the target cells. We should ask if the cytotoxicity is reproduced in cases where lung-derived cells other than fibroblasts, such as alveolar epithelial cells, are used as the target cells instead.

The secretion of MMP-9, a critical cytotoxic factor of NETs ${ }^{40}$, from PMA-stimulated neutrophils occurred equivalently under hypoxic and normoxic conditions. Clinical studies have demonstrated increased MMP9 levels in the sputum and plasma of COPD patients, and that the levels are correlated with disease severity ${ }^{43-45}$. Likewise, increased MMP-9 in the plasma of PAH patients and its involvement in the progression of disease has been reported ${ }^{46}$. Various factors other than MMP-9 released from NETforming neutrophils, such as cell-free histones ${ }^{29}$, could also contribute to the NET-mediated cytotoxicity.

It remains to be determined whether round NETs are generated in the lung tissues with pneumonia, and those generated under hypoxia can trap and eliminate pneumonia pathogens. However, it is not expected that round NETs will trap microorganisms as effectively as lytic NETs, which constitute extracellular weblike DNA. In patients with preexisting lung diseases, it is possible that neutrophils are not able to fight sufficiently against pneumonia pathogens due to the hypoxic circumstances. In addition, diverse inflammatory factors, including chemical mediators and cytokines, produced in the lungs of patients with pneumonia are also able to affect the preexisting lung diseases. 
In summary, when patients with preexisting lung diseases develop pneumonia, tissue hypoxia progresses in the lungs, and incompletely formed NETs, which are resistant to degradation by DNase I and which possess cytotoxic potential, are generated in the tissue. Accumulation of neutrophils that form degradation-resistant NETs and possess cytotoxic potential, including secretion of MMP-9, are expected to be involved, at least in part, in exacerbation of the underlying lung diseases. Even so, there is no doubt that NETs themselves are required to eliminate pathogens of pneumonia. Therefore, a strategy to make neutrophils release proper NETs that are then degraded adequately after accomplishment of their roles seems to be an important next subject of research.

\section{Declarations}

\section{Author Contributions}

S.M., I.T., and A.I. designed research. S.M., K.K., and M.I. performed research. S.M., Y.N., A.S., D.N., S.T., U.T., I.T., and A.I. analyzed data. S.M. and A.I. wrote the paper. All authors discussed the results and contributed to the preparation of the manuscript.

\section{Competing of interest}

The authors declare no competing interests.

\section{References}

1. Ruuskanen, O., et al. Viral pneumonia. Lancet. 377: 1264-1275 (2011).

2. Fein, A. M. Pneumonia in the elderly. Special diagnostic and therapeutic considerations. Med Clin North Am. 78: 1015-1033 (1994).

3. Woodhead, M., et al. Guidelines for the management of adult lower respiratory tract infections. Eur Respir J. 26: 1138-1180 (2005).

4. Seemungal, T., et al. Respiratory viruses, symptoms, and inflammatory markers in acute exacerbations and stable chronic obstructive pulmonary disease. Am J Respir Crit Care Med. 164: 1618-1623 (2001).

5. Rich, S., et al. Primary pulmonary hypertension. A national prospective study. Ann Intern Med. 107: 216-223, (1987).

6. Brinkmann, V., et al. Neutrophil extracellular traps kill bacteria. Science. 303: 1532-1535 (2004).

7. Hakkim, A., et al. Activation of the Raf-MEK-ERK pathway is required for neutrophil extracellular trap formation. Nat Chem Biol. 7: 75-77 (2011).

8. Kaplan, M. J., et al. Neutrophil extracellular traps: double-edged swords of innate immunity. $J$ Immunol. 189: 2689-2695 (2012).

9. Castanheira, F. V. S., et al. Neutrophils and NETs in modulating acute and chronic inflammation. Blood. 133: 2178-2185 (2019). 
10. Wang, Y., et al. Histone hypercitrullination mediates chromatin decondensation and neutrophil extracellular trap formation. J Cell Biol. 184: 205-213 (2009).

11. Li, P., et al. PAD4 is essential for antibacterial innate immunity mediated by neutrophil extracellular traps. J Exp Med. 207: 1853-1862 (2010).

12. Metzler, K. D., et al. A myeloperoxidase-containing complex regulates neutrophil elastase release and actin dynamics during NETosis. Cell Rep. 8: 883-896 (2014).

13. Papayannopoulos, V., et al. Neutrophil elastase and myeloperoxidase regulate the formation of neutrophil extracellular traps. J Cell Biol. 191: 677-691 (2010).

14. Kambara, H., et al. Gasdermin D Exerts Anti-inflammatory Effects by Promoting Neutrophil Death. Cell Rep. 22: 2924-2936 (2018).

15. Sollberger, G., et al. Gasdermin D plays a vital role in the generation of neutrophil extracellular traps. Sci Immunol. 3: eaar6689 (2018).

16. Pedersen, F., et al. Neutrophil extracellular trap formation and extracellular DNA in sputum of stable COPD patients. Respir Med. 109: 1360-1362 (2015).

17. Grabcanovic-Musija, F., et al. Neutrophil extracellular trap (NET) formation characterises stable and exacerbated COPD and correlates with airflow limitation. Respir Res. 16: 59 (2015).

18. Stockley, J. A., et al. Aberrant neutrophil functions in stable chronic obstructive pulmonary disease: the neutrophil as an immunotherapeutic target. Int Immunopharmacol. 17: 1211-1217 (2013).

19. Lugli, E. B., et al. Expression of citrulline and homocitrulline residues in the lungs of non-smokers and smokers: implications for autoimmunity in rheumatoid arthritis. Arthritis Res Ther. 17: 9 (2015).

20. Dicker, A. J., et al. Neutrophil extracellular traps are associated with disease severity and microbiota diversity in patients with chronic obstructive pulmonary disease. J Allergy Clin Immunol. 141: 117127 (2018).

21. Aldabbous, L., et al. Neutrophil Extracellular Traps Promote Angiogenesis: Evidence From Vascular Pathology in Pulmonary Hypertension. Arterioscler Thromb Vasc Biol. 36: 2078-2087 (2016).

22. Saffarzadeh, M., et al. Neutrophil extracellular traps directly induce epithelial and endothelial cell death: a predominant role of histones. PLoS One. 7: e32366 (2012).

23. Branitzki-Heinemann, K., et al. Formation of Neutrophil Extracellular Traps under Low Oxygen Level. Front Immunol. 7: 518 (2016).

24. Ong, C. W. M., et al. Hypoxia increases neutrophil-driven matrix destruction after exposure to Mycobacterium tuberculosis. Sci Rep. 8: 11475 (2018).

25. Hakkim, A., et al. Impairment of neutrophil extracellular trap degradation is associated with lupus nephritis. Proc Natl Acad Sci U S A. 107: 9813-9818 (2010).

26. Fernandez-Torres, J., et al. Hypoxia-Inducible Factors (HIFs) in the articular cartilage: a systematic review. Eur Rev Med Pharmacol Sci. 21: 2800-2810 (2017).

27. Masuda, S., et al. Measurement of NET formation in vitro and in vivo by flow cytometry. Cytometry $A$. 91: 822-829 (2017). 
28. Masuda, S., et al. Formation and Disordered Degradation of Neutrophil Extracellular Traps in Necrotizing Lesions of Anti-Neutrophil Cytoplasmic Antibody-Associated Vasculitis. Am J Pathol. 189: 839-846 (2019).

29. Nakazawa, D., et al. Histones and Neutrophil Extracellular Traps Enhance Tubular Necrosis and Remote Organ Injury in Ischemic AKI. J Am Soc Nephrol. 28: 1753-1768 (2017).

30. Harris, A. J., et al. IL4Ra Signaling Abrogates Hypoxic Neutrophil Survival and Limits Acute Lung Injury Responses In Vivo. Am J Respir Crit Care Med. 200: 235-246 (2019).

31. Takito, J., et al. Symmetrical retrograde actin flow in the actin fusion structure is involved in osteoclast fusion. Biol Open. 6: 1104-1114 (2017).

32. Lin, D. C., et al. Cytochalasins inhibit nuclei-induced actin polymerization by blocking filament elongation. J Cell Biol. 84: 455-460 (1980).

33. Geering, B., et al. Peculiarities of cell death mechanisms in neutrophils. Cell Death Differ. 18: 14571469 (2011).

34. Nakazawa, D., et al. Abnormal conformation and impaired degradation of propylthiouracil-induced neutrophil extracellular traps: implications of disordered neutrophil extracellular traps in a rat model of myeloperoxidase antineutrophil cytoplasmic antibody-associated vasculitis. Arthritis Rheum. 64: 3779-3787 (2012).

35. Stojkov, D., et al. ROS and glutathionylation balance cytoskeletal dynamics in neutrophil extracellular trap formation. J Cell Biol. 216: 4073-4090, (2017).

36. Movitz, C., et al. Quantification of annexin I in subcellular fractions of human neutrophils reveals an exclusive cytosolic localisation. Cell Biol Int. 25: 963-969 (2001).

37. Hoenderdos, K., et al. Hypoxia upregulates neutrophil degranulation and potential for tissue injury. Thorax. 71: 1030-1038 (2016).

38. Kabsch, W., et al. Atomic structure of the actin:DNase I complex. Nature. 347: 37-44 (1990).

39. Leffler, J., et al. Neutrophil extracellular traps that are not degraded in systemic lupus erythematosus activate complement exacerbating the disease. J Immunol. 188: 3522-3531 (2012).

40. Carmona-Rivera, C., et al. Neutrophil extracellular traps induce endothelial dysfunction in systemic lupus erythematosus through the activation of matrix metalloproteinase-2. Ann Rheum Dis. 74: 1417-1424 (2015).

41. Lee, J. W., et al. Hypoxia-inducible factor (HIF-1)a: its protein stability and biological functions. Exp Mol Med. 36: 1-12 (2004).

42. Semenza, G. L. Defining the role of hypoxia-inducible factor 1 in cancer biology and therapeutics. Oncogene. 29: 625-634 (2010).

43. Mercer, P. F., et al. MMP-9, TIMP-1 and inflammatory cells in sputum from COPD patients during exacerbation. Respir Res. 6: 151 (2005).

44. Vernooy, J. H., et al. Increased activity of matrix metalloproteinase-8 and matrix metalloproteinase-9 in induced sputum from patients with COPD. Chest. 126: 1802-1810 (2004). 
45. Koo, H. K., et al. Relationship between plasma matrix metalloproteinase levels, pulmonary function, bronchodilator response, and emphysema severity. Int J Chron Obstruct Pulmon Dis. 11: 1129-1137 (2016).

46. Chelladurai, P., et al. Matrix metalloproteinases and their inhibitors in pulmonary hypertension. Eur Respir J. 40: 766-782 (2012).

\section{Figures}


a

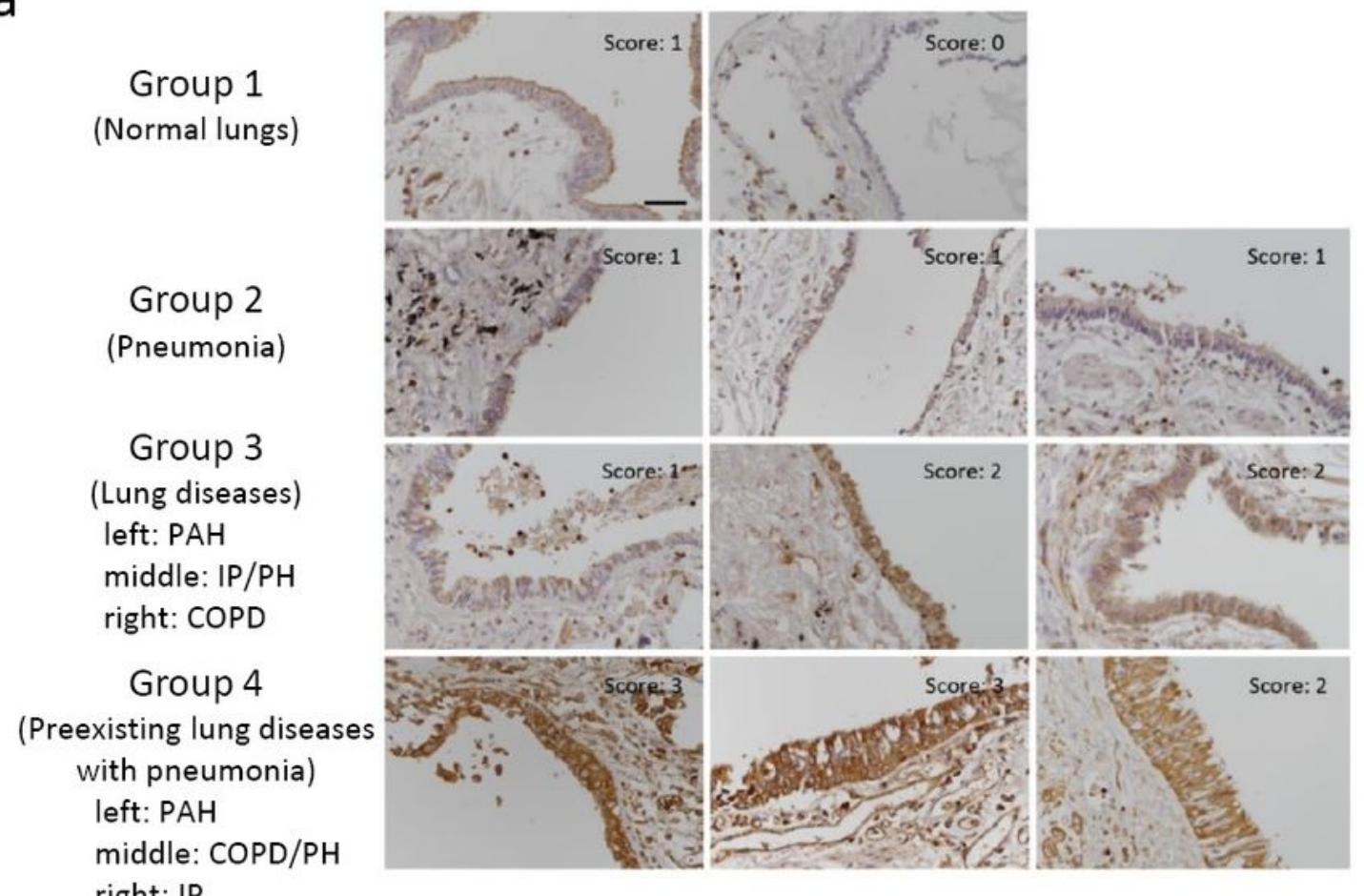

right: IP

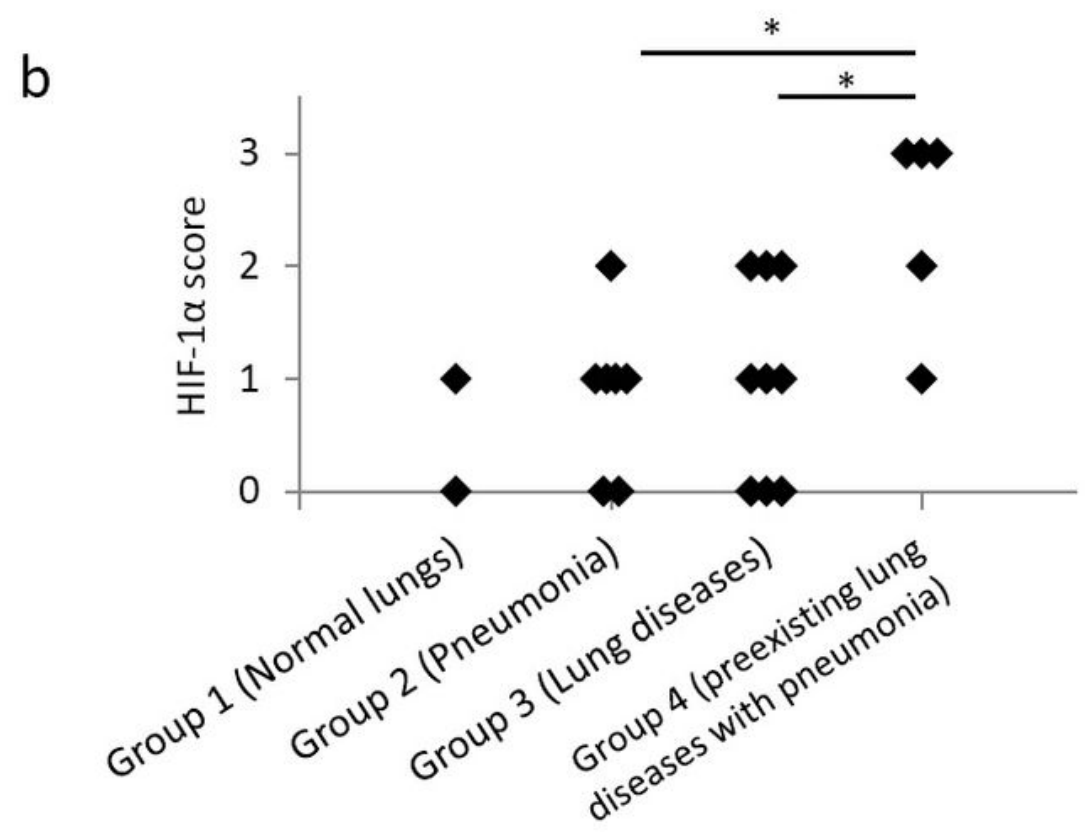

\section{Figure 1}

Expression of HIF-1a in the lungs Expression of HIF-1a in the lungs was assessed by IHC (Group 1, normal lung, n=2; Group 2, pneumonia, n=7; Group 3, lung diseases without pneumonia, n=9; and Group 4, preexisting lung diseases with pneumonia, $n=5$ ). (a) Representative photos with the bronchus are shown. HIF-1a expression levels in bronchial epithelial cells were scored as 0 , undetectable; 1 , weak; 2 , moderate; and 3, strong. PAH, pulmonary arterial hypertension; IP, interstitial pneumonitis; $\mathrm{PH}$, pulmonary 
hypertension; COPD, chronic obstructive pulmonary disease. Bar, $50 \mu \mathrm{m}$. (b) The scores of the 4 groups were plotted and compared. ${ }^{*} p<0.05$.

a

Group 1

(Normal lungs)

Group 2

(Pneumonia)
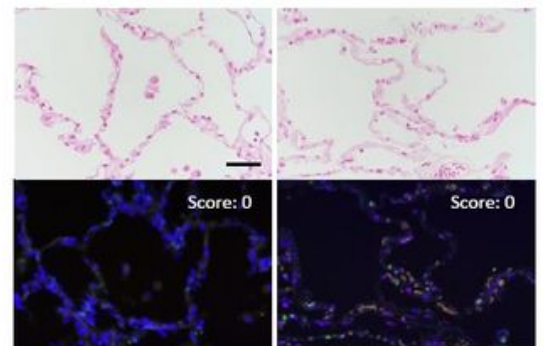

DNA Cit H3 MPO
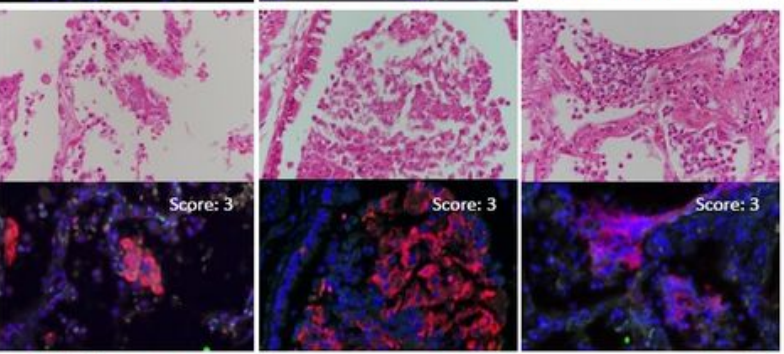

Group 3

(Lung diseases) left: PAH middle: IP/PH right: $C O P D$
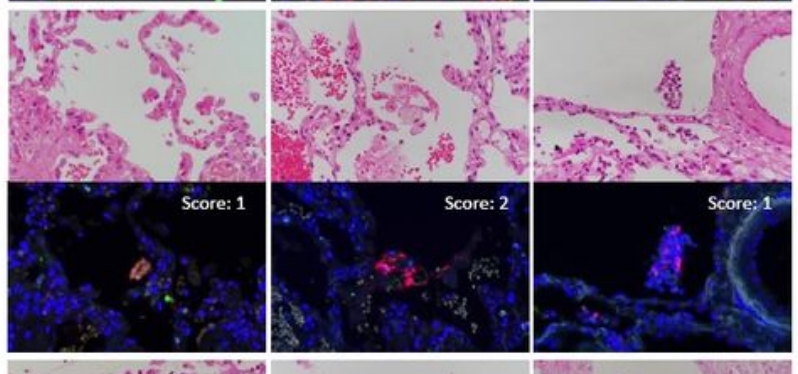

Group 4

(Preexisting lung diseases with pneumonia) left: PAH middle: $\mathrm{COPD} / \mathrm{PH}$ right: IP
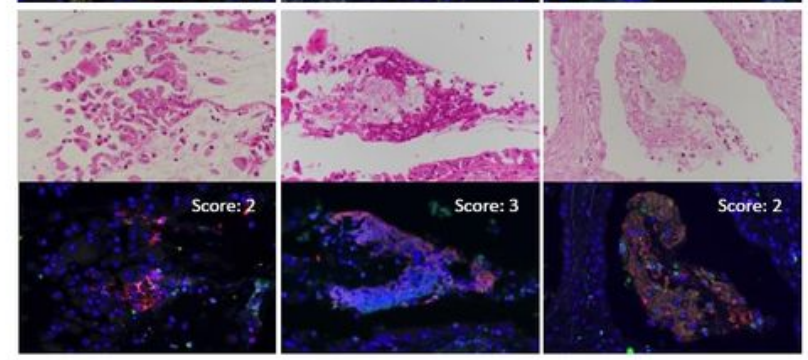

b

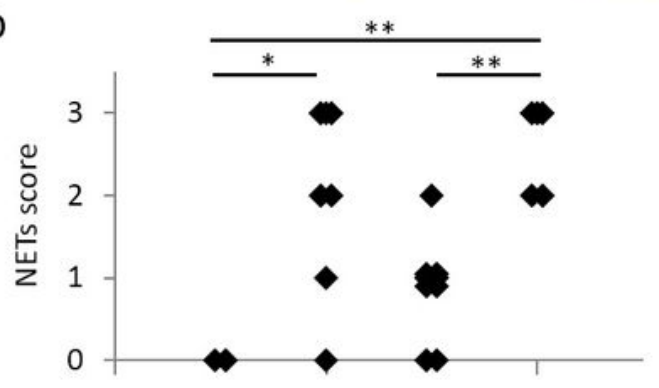

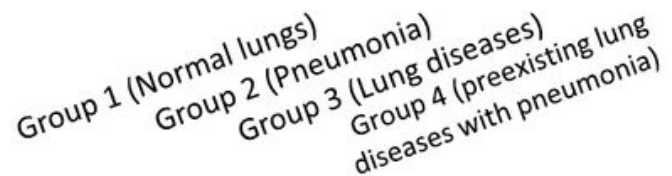

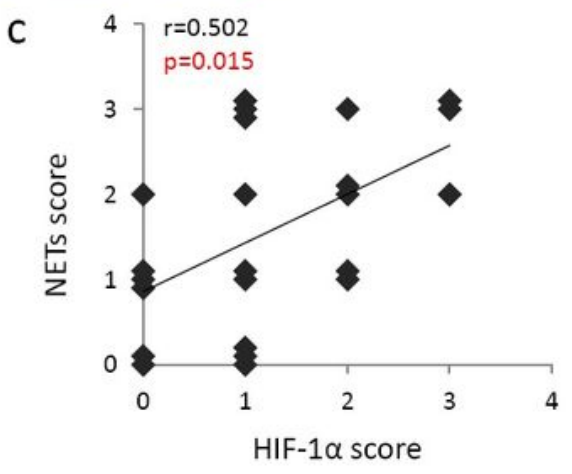

\section{Figure 2}

NETs in the lungs NETs in the lungs were assessed by immunofluorescent staining (Group 1, normal lung, $\mathrm{n}=2$; Group 2, pneumonia, $\mathrm{n}=7$; Group 3, lung diseases without pneumonia, $\mathrm{n}=9$; and Group 4, preexisting lung diseases with pneumonia, $n=5$ ). NETs are recognized as $\mathrm{Cit} H 3+$ substances (red) mixed with DAPI+ 
DNA (blue) and MPO (green). (a) The amount of NETs was semi-quantified as follows: Score 0, unrecognizable; Score 1, scattered small NETs; Score 2, scattered large NETs; and Score 3, dense large NETs. Representative photos are shown. PAH, pulmonary arterial hypertension; IP, interstitial pneumonitis; $\mathrm{PH}$, pulmonary hypertension; COPD, chronic obstructive pulmonary disease. Bar, $50 \mu \mathrm{m}$. (b) The scores of the 4 groups were plotted and compared. ${ }^{*} p<0.05$, $* * p<0.01$. (c) Correlation between HIF-1a expression and amount of NETs.

a
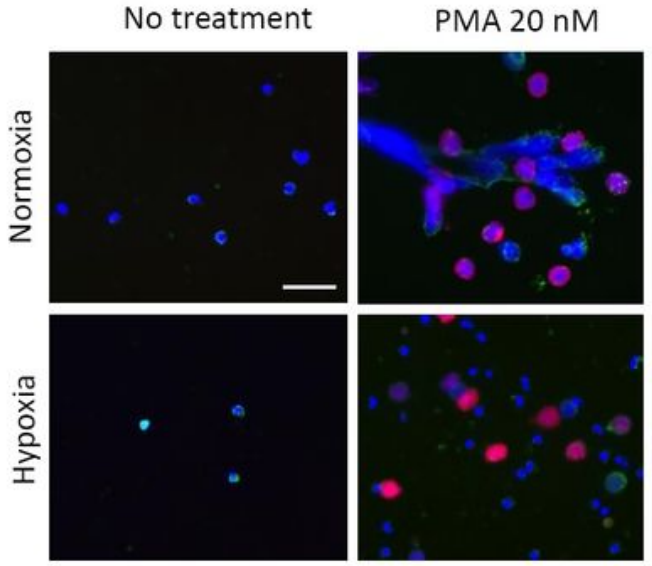

Normoxia

C
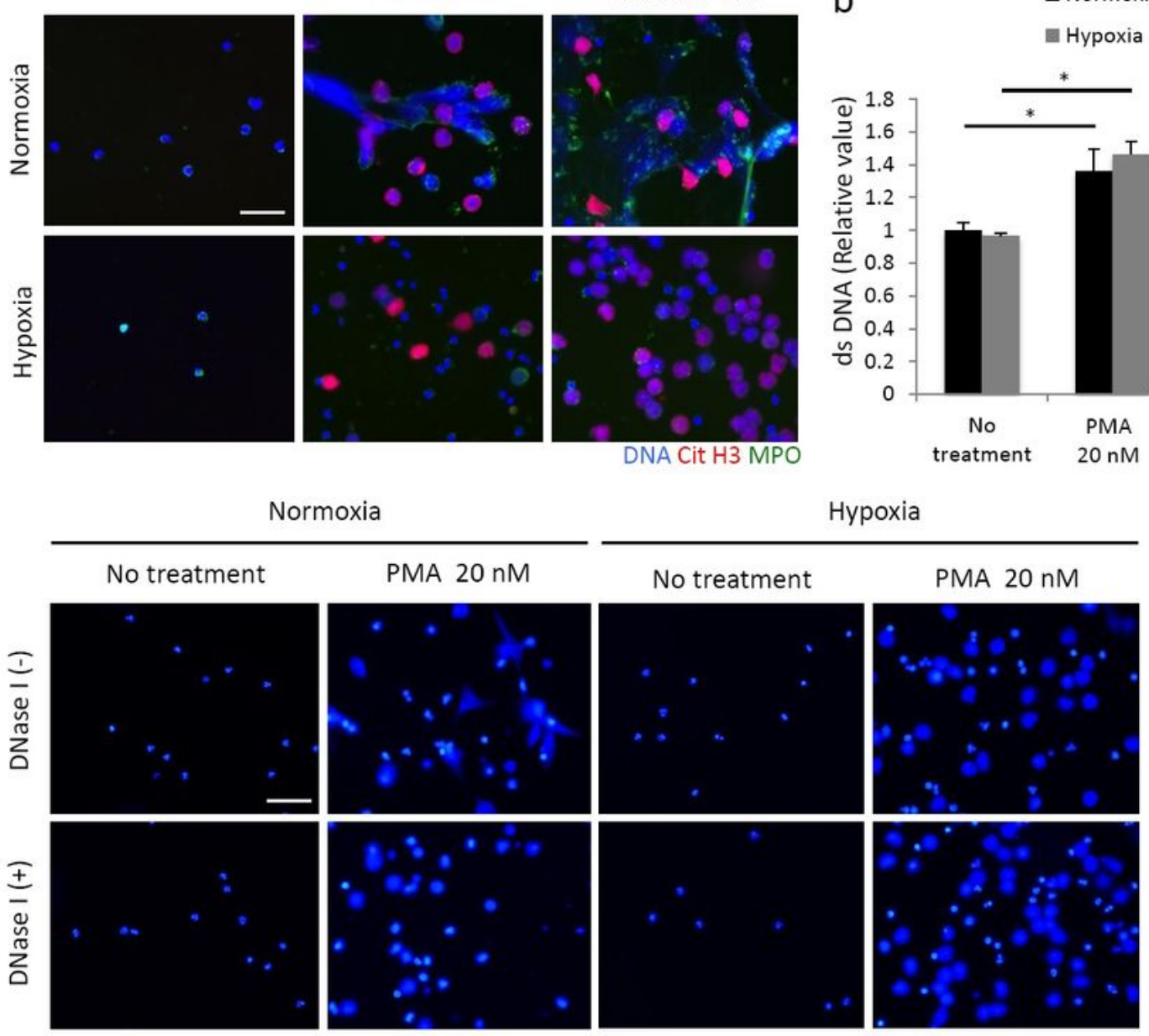

DNA Cit H3 MPO

Hypoxia

d

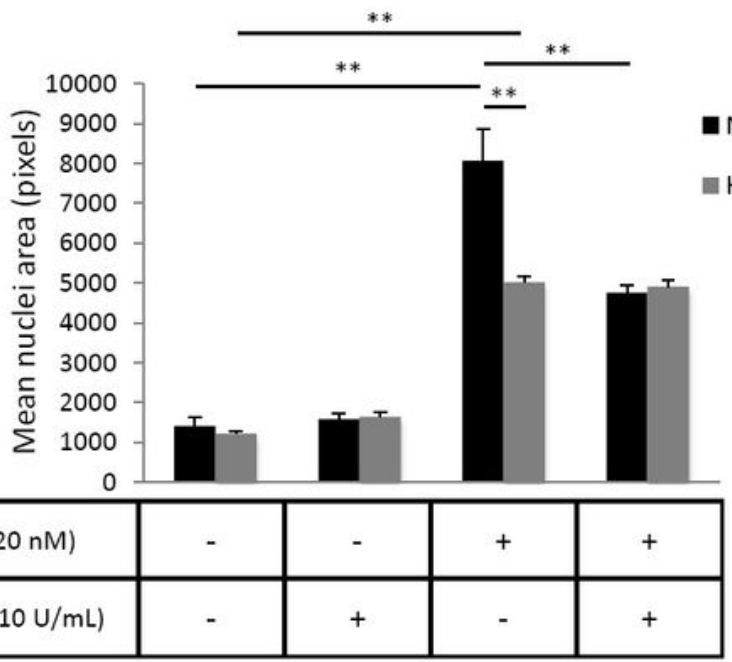

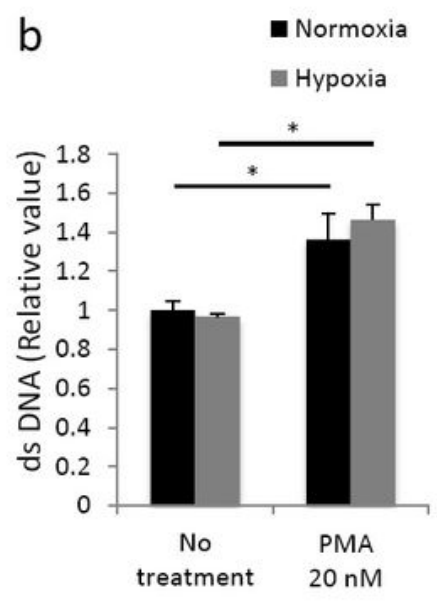

Normoxia

Hypoxia

Figure 3 
NET induction under normoxic and hypoxic conditions (a) Peripheral blood neutrophils were incubated with or without PMA (20 or $100 \mathrm{nM})$ for 4 hours under normoxic $(21 \%$ 02) or hypoxic $(1 \% 02)$ conditions. Thereafter, immunofluorescent staining for NETs was conducted. Representative photos are shown. Blue, DNA; Red, Cit H3; and Green, MPO. Bar, $50 \mu \mathrm{m}$. (b) Concentrations of dsDNA in the supernatants. * $\mathrm{p}<$ 0.05. (c) After 4 hour incubation of neutrophils with PMA (20 nM) under normoxic (21\% 02) or hypoxic $(1 \% 02)$ conditions, the cells were reacted with DNase I $(10 \mathrm{U} / \mathrm{mL})$ and incubated for a further 30 minutes. Representative photos are shown. Blue: DNA. Bar, $50 \mu \mathrm{m}$. (d) Five photographs were taken randomly before and after DNase I treatment. Mean nuclei area was calculated using Image J 1.50i software as follows: DAPI+ areas/number of neutrophils (pixels). ${ }^{\star \star} p<0.01$. 
a

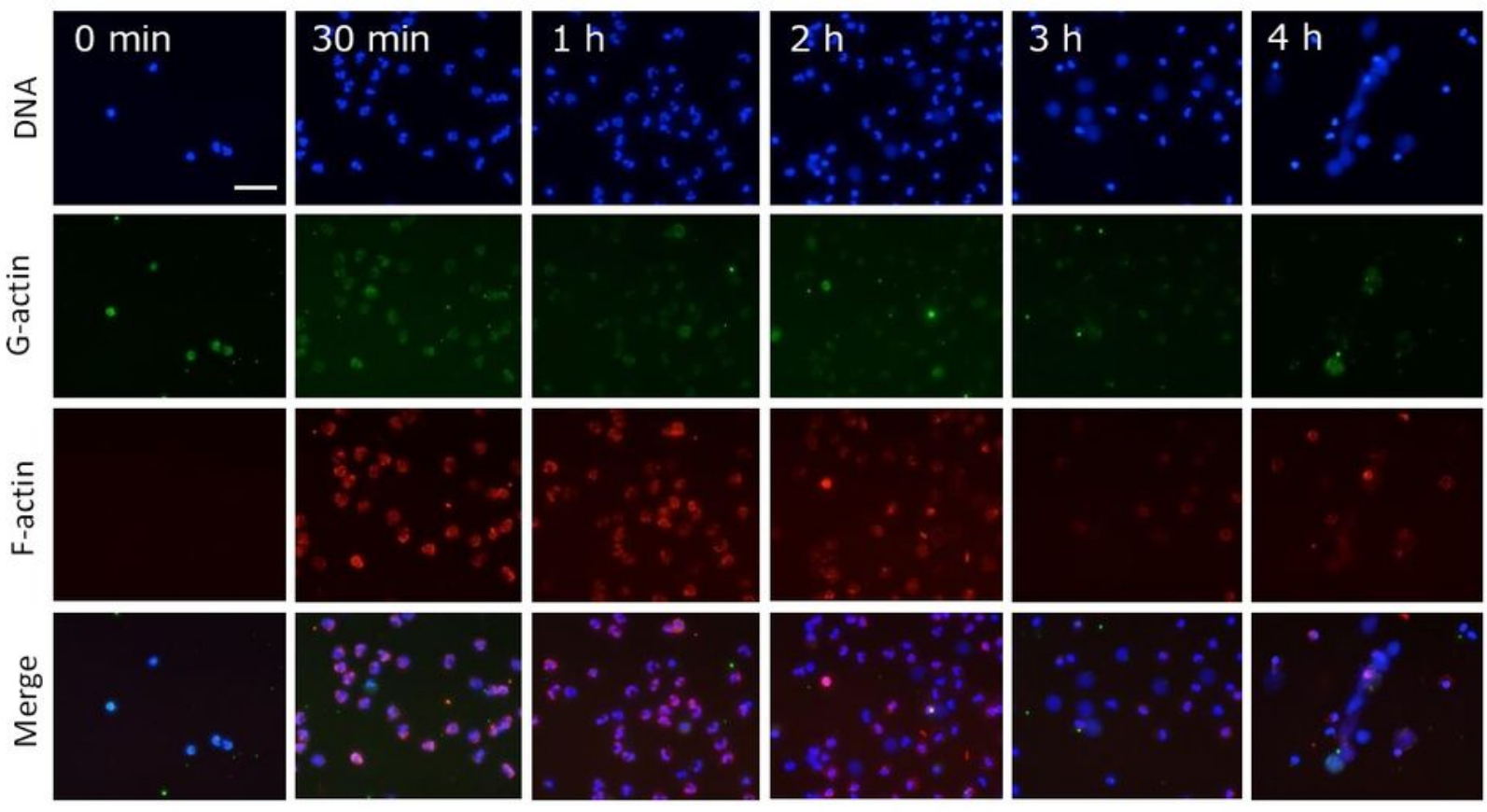

b

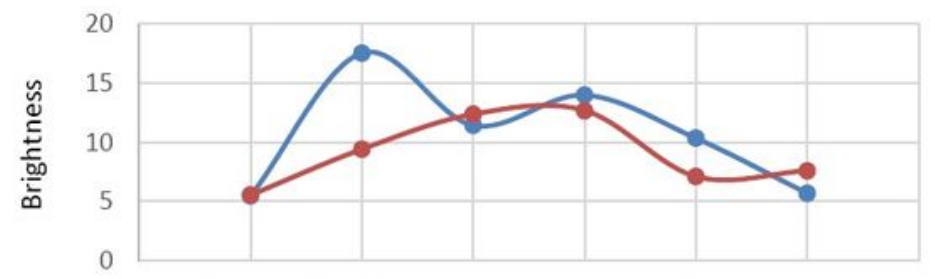

Time after stimulation: $0 \mathrm{~min} \quad 30 \mathrm{~min} \quad 1 \mathrm{~h} \quad 2 \mathrm{~h} \quad 3 \mathrm{~h} \quad 4 \mathrm{~h}$

$\rightarrow$ Green $\rightarrow$ Red

C

Normoxia

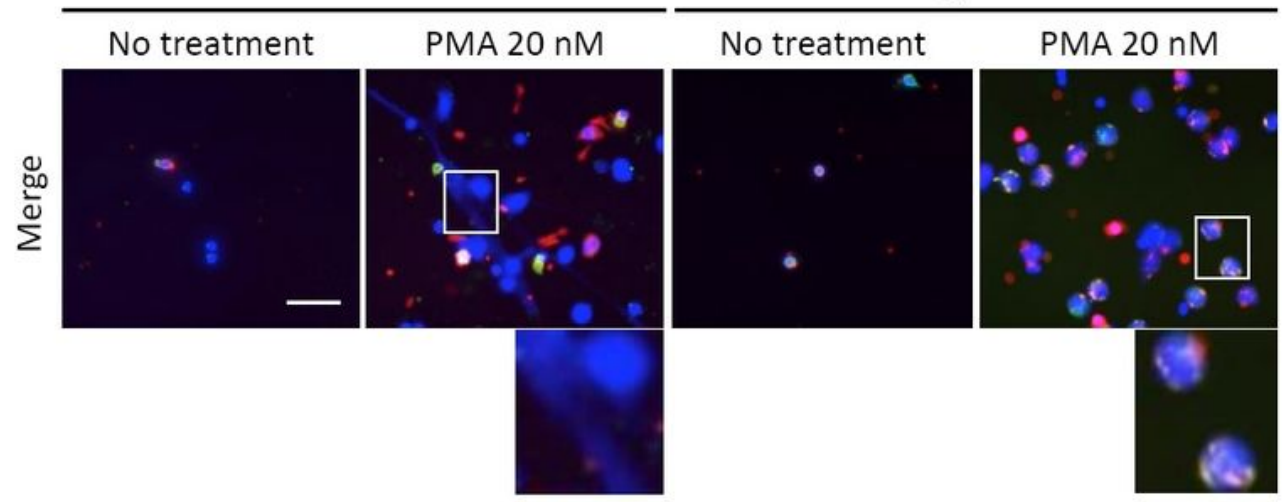

DNA

G-actin

F-actin

\section{Figure 4}

Actin rearrangement during NET formation under normoxic and hypoxic conditions (a) To follow chronological actin rearrangement during NET formation, peripheral blood neutrophils were incubated with PMA (20 nM) for 4 hours under normoxic conditions (21\% O2). Fluorescent staining for G- and Factins was carried out at 0, 0.5, 1, 2, 3, and 4 hours after addition of PMA. (b) Brightness of green and red fluorescence that represents the expression of G-actin and F-actin, respectively, was measured by Adobe 
Photoshop Elements 14 software. (c) To compare actin distribution during NET formation, peripheral blood neutrophils were incubated with or without PMA $(20 \mathrm{nM})$ for 4 hours under normoxic $(21 \% 02)$ or hypoxic ( $1 \%$ O2) conditions. After that, fluorescent staining for $\mathrm{G}$ - and F-actins was carried out. Representative photos are shown. Blue, DNA; Green, G-actin; and Red, F-actin. Bar, $50 \mu \mathrm{m}$.

a

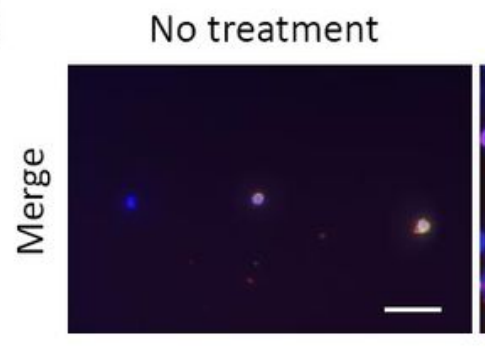

b
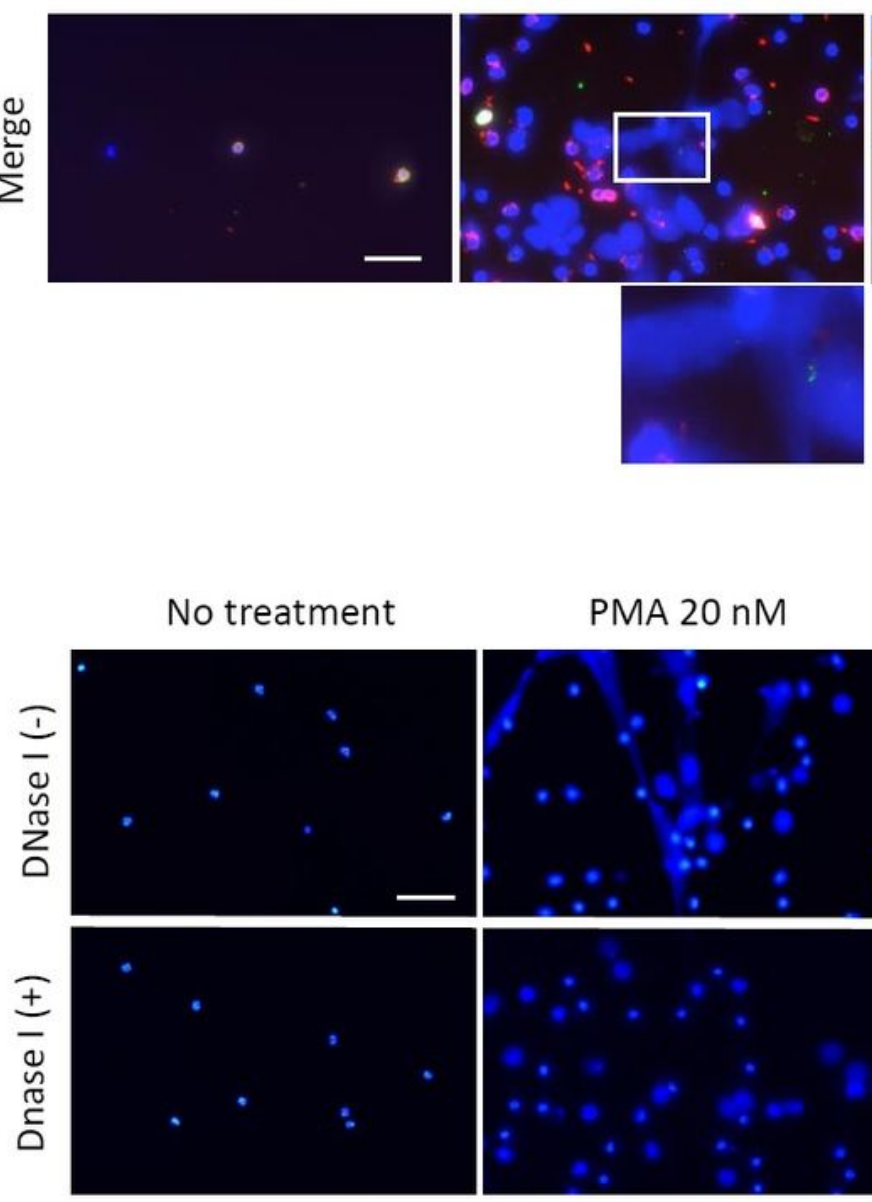

PMA $20 \mathrm{nM}+$ CyD $0.5 \mu \mathrm{M}$

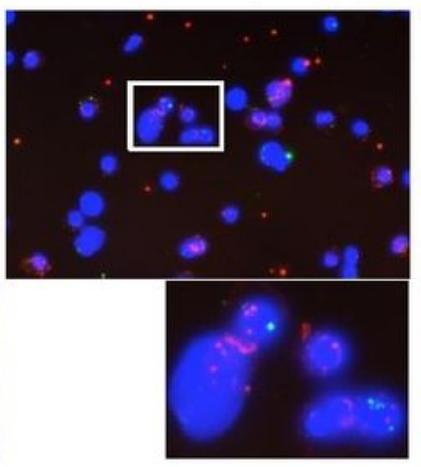

DNA

G-actin

F-actin

C

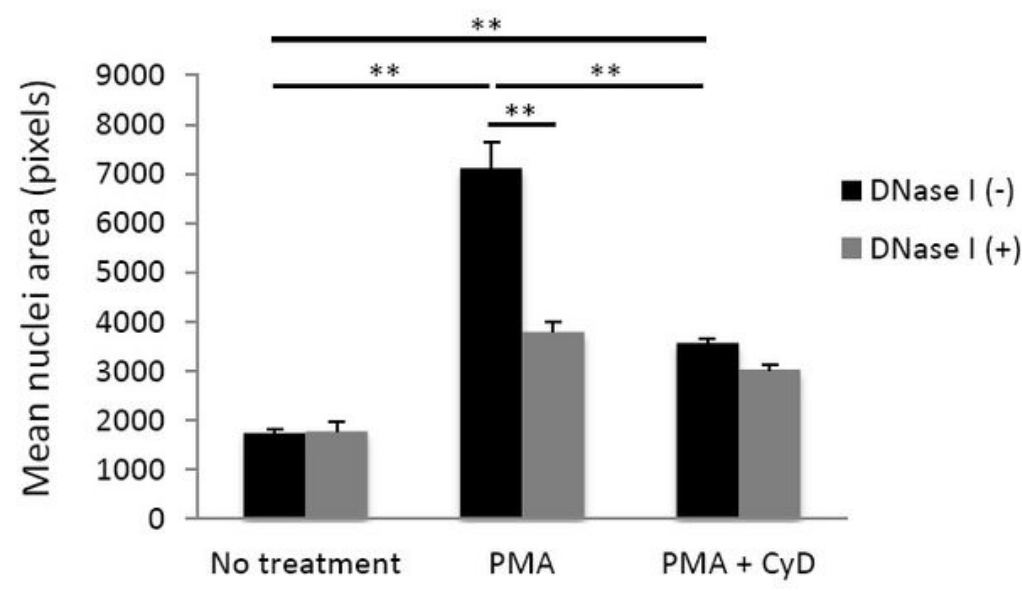

Figure 5 
Effects of inhibition of actin polymerization on susceptibility of NETs to DNase I (a) Peripheral blood neutrophils were exposed to PMA $(20 \mathrm{nM})$ with or without CyD $(0.5 \mu \mathrm{M})$ for 4 hours under normoxic conditions (21\% 02). Thereafter, fluorescent staining for G-actin (green) and F-actin (red) was conducted. Representative photos are shown. Blue, DNA. Bar, $50 \mu \mathrm{m}$. (b) After 4 hours incubation of neutrophils with PMA $(20 \mathrm{nM})$ with or without CyD $(0.5 \mu \mathrm{M})$ under normoxic conditions $(21 \% 02)$, the cells were reacted with DNase I $(10 \mathrm{U} / \mathrm{mL})$ and were then incubated for a further 30 minutes. Representative photos are shown. Blue: DNA. Bar, $50 \mu \mathrm{m}$. (c) Five photographs were taken randomly. Mean nuclei area (pixels) was calculated using Image J 1.50i software. ${ }^{* *} \mathrm{p}<0.01$. 
a

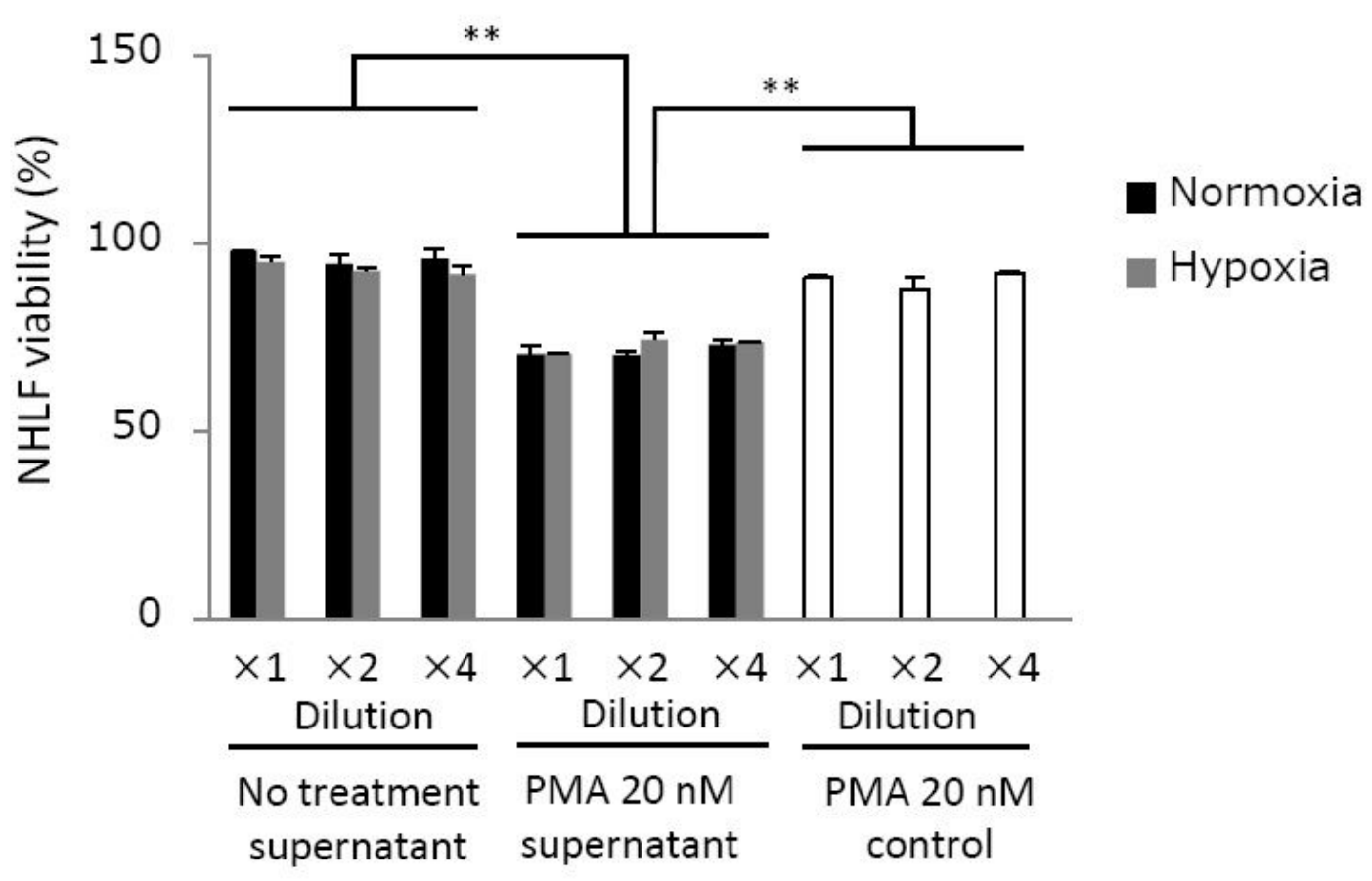

b

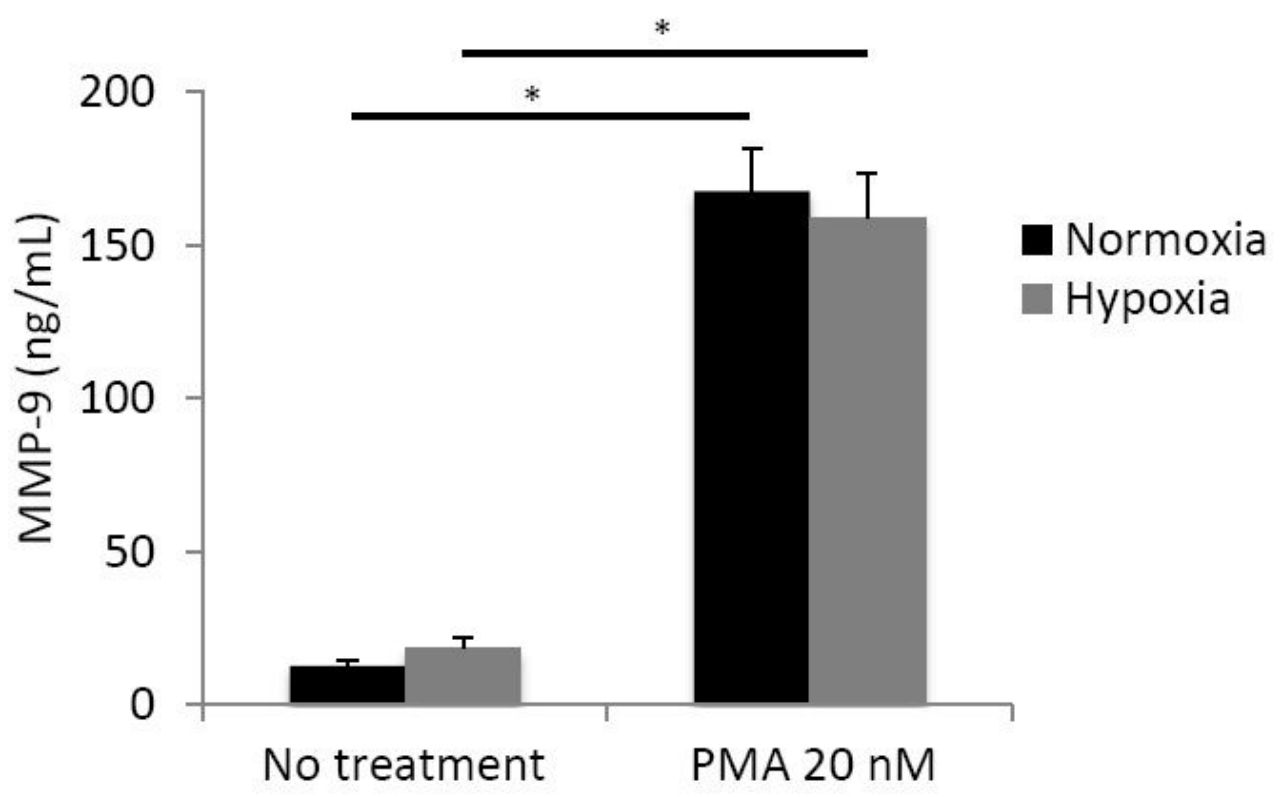

Figure 6

Cytotoxicity of neutrophils stimulated under hypoxic conditions (a) NHLF were incubated with 1:1, 1:2, and 1:4 diluted supernatants of neutrophils stimulated under normoxic $(21 \% 02)$ and hypoxic $(1 \% 02)$ conditions. Alternatively, NHLF were incubated with medium that contained equivalent amounts of PMA (white columns). Twenty-four hours later, viability of NHLF was calculated using a cell counting kit. (b) The concentration of MMP-9 in the supernatants was determined by ELISA. ${ }^{*} p<0.05,{ }^{*} \mathrm{p}<0.01$. 


\section{Supplementary Files}

This is a list of supplementary files associated with this preprint. Click to download.

- FigureS1.pdf

- Figures2.pdf

- FigureS3.pdf

- FigureS4.pdf

- Tables1.docx 